\title{
Manifestaciones neurológicas graves de la gripe durante la temporada gripal 2018-2019: serie de casos de 13 pacientes pediátricos
}

Severe neurological manifestations of influenza during 2018-2019 influenza season: Case series of 13 pediatric patients

\author{
Dr. M. Gultekin Kutluk y Dra. E. Naz Kadem ${ }^{b}$
}

\section{RESUMEN}

La gripe se asocia al aparato respiratorio, especialmente en invierno, y puede causar complicaciones neurológicas. Se evaluó a pacientes pediátricos con manifestaciones neurológicas graves por gripe desde septiembre de 2018 hasta febrero de 2019 para determinar características clínicas, neuroimagenología, tratamiento y resultados. El objetivo fue evaluar la encefalitis asociada a la gripe y destacar diferentes manifestaciones neurológicas y cambios de neuroimagenología. El estudio incluyó a 13 pacientes. Los síntomas neurológicos ocurrieron tras los síntomas típicos de la gripe. Los cambios de neuroimagenología incluyen alteraciones de señal de la sustancia blanca cortical y subcortical, edema localizado o generalizado y lesiones multifocales simétricas bilaterales en el tálamo y la médula del cerebelo. Las opciones terapéuticas incluyen metilprednisolona en inyección intravenosa, inmunoglobulina intravenosa, plasmaféresis y oseltamivir. Es fundamental considerar la encefalitis asociada a la gripe en pacientes con convulsiones, la encefalopatía con hallazgos radiológicos compatibles, e iniciar el tratamiento lo antes posible.

Palabras clave: encefalitis, encefalopatía, sindrome de GuillainBarré, cuerpo calloso, pediatría.

http: / / dx.doi.org/10.5546/ aap.2021.e142

Texto completo en inglés:

http: / / dx.doi.org/ 10.5546/ aap.2021.eng.e142

Cómo citar: Kutluk MG, Kadem EN. Manifestaciones neurológicas graves de la gripe durante la temporada gripal 2018-2019: serie de casos de 13 pacientes pediátricos. Arch Argent Pediatr 2021;119(2):e142-e148.

a. Departamento de Neurología Pediátrica.

b. Consultorios de Pediatría.

Hospital de Formación e Investigación de Antalya, Antalya, Turquía.

\section{Correspondencia:}

Dr. M. Gultekin Kutluk: gultekinkutluk@gmail.com

Financiamiento: Ninguno.

Conflicto de intereses: Ninguno que declarar.

Recibido: 20-5-2020

Aceptado: 23-10-2020

\section{GLOSARIO}

ADC: Coeficiente de difusión aparente.

EEG: Electroencefalografía.

FLAIR: Recuperación de la inversión atenuada por líquidos.

GOSE: Escala de resultados de Glasgow

ampliada.

IgIV: Inmunoglobulina intravenosa.

LCR: Líquido cefalorraquídeo.

MP: Metilprednisolona.

PDRG: Pruebas de diagnóstico rápido de la gripe.

RM: Resonancia magnética.

\section{INTRODUCCIÓN}

Los niños generalmente tienen infecciones gripales con síntomas respiratorios y son hospitalizados por complicaciones respiratorias y neurológicas, sobre todo en invierno. A nivel mundial, casi 90 millones de niños padecen infecciones gripales cada año. ${ }^{1,2}$ Se observan diferencias en la incidencia de las complicaciones neurológicas asociadas a la gripe según la región; Glaser y cols., informaron complicaciones neurológicas en 1,2/1 000 000. En Japón se informó una incidencia de encefalitis asociada a la gripe de 6-11/1 000000 en 1999-2000. ${ }^{3}$ Las manifestaciones neurológicas tienen un amplio espectro de gravedad y se caracterizan a partir de los hallazgos de la resonancia magnética (RM). Además, hay muchos casos con diferentes alteraciones en la RM que no son frecuentes en la bibliografía. ${ }^{2}$ En este estudio se presentan 13 casos pediátricos con encefalitis asociada a la gripe durante la temporada gripal 2018-2019 en un único centro. Además, se señalan las diferentes características de gravedad y neuroimagenología de la encefalitis asociada a la gripe y las opciones terapéuticas.

\section{MÉTODOS \\ Población del estudio}

Se llevó a cabo una investigación retrospectiva con pacientes de entre 6 meses 
y 18 años de edad que habían sido ingresados en un único centro por infecciones de gripe $\mathrm{A}$ con complicaciones neurológicas graves entre septiembre de 2018 y diciembre de 2019. E1 consentimiento firmado se obtuvo de los tutores legales de los participantes.

\section{Estudios virológicos}

Se realizaron una reacción en cadena de la polimerasa con retrotranscripción en tiempo real (RT-PCR) y pruebas de diagnóstico rápido de la gripe (PDRG) con las muestras de las vías respiratorias y del líquido cefalorraquídeo (LCR).

\section{Estudios neuroimagenológicos y electrofisiológicos}

Se realizó una electroencefalografía (EEG) a todos los pacientes, las cuales fueron evaluadas por el mismo neurólogo pediátrico. Las imágenes de RM ponderadas en T1 y T2, la recuperación de la inversión atenuada por líquidos (FLAIR), las imágenes ponderadas por difusión (DWI) y el

TABLA 1. Datos demográficos, primeras características de presentación y evolución clínica, resultado de los pacientes con encefalitis asociada a la gripe

\begin{tabular}{|c|c|c|c|c|c|c|c|c|c|}
\hline $\begin{array}{l}\text { Número } \\
\text { de caso }\end{array}$ & $\begin{array}{l}\text { Edad/ } \\
\text { Sexo }\end{array}$ & $\begin{array}{c}\text { Inicio de } \\
\text { los síntomas } \\
\text { respiratorios (días) }\end{array}$ & $\begin{array}{l}\text { Presentación } \\
\text { clínica }\end{array}$ & $\begin{array}{l}\text { GCS en la } \\
\text { primera } \\
\text { consulta }\end{array}$ & Vacuna & $\begin{array}{c}\text { EEG } \\
\text { la UCIP }\end{array}$ & $\begin{array}{l}\text { Días en } \\
\text { (tras el } \\
\text { amiento) }\end{array}$ & GOSE & $\begin{array}{c}\text { Secuelas } \\
\text { neurológicas }\end{array}$ \\
\hline 1 & $21 \mathrm{~m} / \mathrm{F}$ & 7 & $\begin{array}{c}\text { Fiebre, rinitis, } \\
\text { convulsiones, estado } \\
\text { mental alterado }\end{array}$ & 10 & $\begin{array}{c}\text { Sin } \\
\text { vacuna }\end{array}$ & Encefalopatía & 8 & 8 & Ninguna \\
\hline 2 & $11 \mathrm{a} / \mathrm{F}$ & 15 & $\begin{array}{l}\text { Disfagia, afasia, } \\
\text { estado mental alterado }\end{array}$ & 12 & $\begin{array}{c}\text { Sin } \\
\text { vacuna }\end{array}$ & Encefalopatía & 16 & 7 & Ninguna \\
\hline 3 & $8 \mathrm{a} / \mathrm{M}$ & 10 & $\begin{array}{l}\text { Disfagia, afasia, } \\
\text { estado mental alterado }\end{array}$ & 13 & $\begin{array}{c}\text { Sin } \\
\text { vacuna }\end{array}$ & Encefalopatía & 17 & 8 & Ninguna \\
\hline 4 & $7 \mathrm{a} / \mathrm{F}$ & 4 & $\begin{array}{c}\text { Pérdida temporal } \\
\text { de la visión }\end{array}$ & 15 & $\begin{array}{c}\text { Sin } \\
\text { vacuna }\end{array}$ & No se realizó & 0 & 8 & Ninguna \\
\hline 5 & $16 \mathrm{a} / \mathrm{M}$ & 6 & $\begin{array}{l}\text { Convulsiones, estado } \\
\text { mental alterado }\end{array}$ & 11 & $\begin{array}{c}\text { Sin } \\
\text { vacuna }\end{array}$ & Encefalopatía & 23 & 6 & $\begin{array}{l}\text { Epilepsia y } \\
\text { trastorno de } \\
\text { la conducta } \\
\end{array}$ \\
\hline 6 & $8 \mathrm{a} / \mathrm{M}$ & 4 & $\begin{array}{l}\text { Convulsiones, estado } \\
\text { mental alterado }\end{array}$ & 12 & $\begin{array}{c}\text { Sin } \\
\text { vacuna }\end{array}$ & Encefalopatía & 4 & 8 & Ninguna \\
\hline 7 & $12 \mathrm{a} / \mathrm{F}$ & 5 & $\begin{array}{c}\text { Cefalea, afasia, } \\
\text { convulsiones, estado } \\
\text { mental alterado }\end{array}$ & 13 & $\begin{array}{c}\text { Sin } \\
\text { vacuna }\end{array}$ & Encefalopatía & 11 & 8 & Ninguna \\
\hline$\overline{8}$ & $10 \mathrm{a} / \mathrm{M}$ & 4 & $\begin{array}{l}\text { Convulsiones, estado } \\
\text { mental alterado }\end{array}$ & 12 & $\begin{array}{c}\text { Sin } \\
\text { vacuna }\end{array}$ & Encefalopatía & 3 & 8 & Ninguna \\
\hline 9 & $18 \mathrm{~m} / \mathrm{M}$ & 2 & $\begin{array}{c}\text { Fiebre, rinitis, } \\
\text { convulsiones, estado } \\
\text { mental alterado }\end{array}$ & 6 & $\begin{array}{c}\text { Sin } \\
\text { vacuna }\end{array}$ & $\begin{array}{c}\text { Estado } \\
\text { convulsivo }\end{array}$ & 5 & 8 & Ninguna \\
\hline 10 & $6 \mathrm{a} / \mathrm{M}$ & 15 & $\begin{array}{l}\text { Distonía, afasia, } \\
\text { síncope }\end{array}$ & 9 & $\begin{array}{c}\text { Sin } \\
\text { vacuna }\end{array}$ & Encefalopatía & 20 & 6 & $\begin{array}{l}\text { Discapacidad } \\
\text { intelectual, } \\
\text { trastorno de } \\
\text { la conducta, } \\
\text { dificultad } \\
\text { para caminar }\end{array}$ \\
\hline 11 & $\begin{array}{c}16 \mathrm{a} / \mathrm{F} \\
{[}\end{array}$ & 11 & $\begin{array}{l}\text { Distonía, afasia, } \\
\text { síncope }\end{array}$ & 8 & $\begin{array}{c}\text { Sin } \\
\text { vacuna }\end{array}$ & $\begin{array}{c}\text { Encefalopatía } \\
\text {, actividad } \\
\text { epiléptica } \\
\text { centrotemporal }\end{array}$ & 44 & 5 & $\begin{array}{l}\text { Discapacidad } \\
\text { intelectual, } \\
\text { tetraparesia } \\
\text { espástica } \\
\end{array}$ \\
\hline 12 & $9 \mathrm{a} / \mathrm{M}$ & 7 & $\begin{array}{l}\text { Cuadro confusional } \\
\text { agudo, estado } \\
\text { mental alterado }\end{array}$ & 9 & $\begin{array}{c}\text { Sin } \\
\text { vacuna }\end{array}$ & Encefalopatía & 29 & 8 & Ninguna \\
\hline$\overline{13}$ & $8 \mathrm{a} / \mathrm{F}$ & 15 & Ataxia & 15 & $\begin{array}{c}\text { Sin } \\
\text { vacuna }\end{array}$ & No se realizó & 0 & 8 & Ninguna \\
\hline
\end{tabular}

GCS: escala de coma de Glasgow; UCIP: unidad de cuidados intensivos pediátricos; GOSE: escala de resultados de Glasgow ampliada; F: femenino; M: masculino. 
coeficiente de difusión aparente $(\mathrm{ACD}, \mathrm{b}=1000)$ se obtuvieron con aparatos de RM de 1,5 T o $3 \mathrm{~T}$. Se utilizó un medio de contraste de gadolinio para demostrar la presencia de inflamación.

\section{Evaluación clínica}

Se evaluó y trató a todos los pacientes en los departamentos de Neurología Pediátrica y de Enfermedades Infecciosas Pediátricas y la Unidad de Cuidados Intensivos Pediátricos (UCIP). Se utilizó la escala de coma de Glasgow (GCS) para describir el nivel general de conciencia. ${ }^{4}$ Se usó la escala de resultados de Glasgow ampliada (GOSE) para determinar el resultado al momento del alta hospitalaria. ${ }^{5}$

\section{RESULTADOS}

\section{Datos demográficos}

Se incluyó a 13 pacientes en el estudio. Los datos demográficos de todos los pacientes se describen en la Tabla 1. Antes de acudir al centro, los pacientes 5, 9 y 11 tenían comorbilidades; los demás estaban sanos. El paciente 5 tenía epilepsia y la paciente 11, retraso mental. El paciente 9 había nacido prematuramente. Tres pacientes tenían padres consanguíneos.

\section{Síntomas neurológicos}

Todos los pacientes presentaron síntomas neurológicos luego de una media de 8,07 días (mín.: 4 días-máx.: 15 días) de síntomas gripales.
El primer síntoma fue el estado alterado de conciencia en 11 de 13 pacientes, y la media de la GCS fue 11,15 (mín.: 6-máx.: 15). Cuatro de ellos recobraron el conocimiento luego del día 1, mientras que 7 tuvieron un deterioro persistente de las funciones corticales superiores. Una paciente tuvo retención urinaria, que se consideró una disfunción neurovegetativa (P2). Seis pacientes tuvieron convulsiones y el paciente 9, estado epiléptico (Tabla 1).

\section{Hallazgos de laboratorio}

En el cultivo de exudado nasal, se detectó gripe A mediante PCR (10 pacientes, 76,9\%) o PDRG (3 pacientes, 23,1\%). No se detectaron microorganismos en las muestras de sangre ni en las de LCR. No se halló ninguna otra alteración en los análisis de laboratorio en relación con las características clínicas mencionadas (Tabla 2).

\section{Hallazgos del estudio neuroimagenológico y de la EEG}

Los resultados anormales de la EEG se informaron como encefalopatía, actividad epiléptica centrotemporal y estado convulsivo (Tabla 1). El paciente 5 presentaba actividad epiléptica frontotemporal durante el seguimiento y la paciente 11, actividad epiléptica centrotemporal en la EEG. En la RM craneal de la paciente 4 , se observaron alteraciones de la señal en el esplenio del cuerpo calloso, que era

TABLA 2. Hallazgos de laboratorio

\begin{tabular}{lcccc}
\hline $\begin{array}{l}\text { Número } \\
\text { de caso }\end{array}$ & LCR & Tipo de gripe & Hemocultivo & Pruebas de encefalitis autoinmunitaria \\
\hline 1 & Normal & Gripe A (PCR) & Estéril & - \\
2 & Normal & Gripe A (H1N1) (PCR) & Estéril & Normal \\
3 & Pleocitosis & Gripe A (H1N1) (PCR) & Estéril & Normal \\
4 & Normal & Gripe A (H1N1) (PDRG) & Estéril & - \\
5 & Normal & Gripe A (PCR) & Estéril & - \\
6 & Normal & Gripe A (PCR) & Estéril & - \\
7 & Normal & Gripe A (PCR) & Estéril & - \\
8 & Normal & Gripe A (PDRG) & Estéril & Normal \\
9 & Normal & Gripe A (PDRG) & Estéril & Normal \\
10 & Normal & Gripe A (PCR) & Estéril & Normal \\
11 & Normal & Gripe A (PCR) & Estéril & - \\
12 & Normal & Gripe A (PCR) & Estéril & Estéril \\
13 & Disociación albumino-citológica & Gripe A (PCR) & . & \\
\hline
\end{tabular}

Pruebas de encefalitis autoinmunitaria: receptor de N-metil-D-aspartato (NMDA), ácido $\alpha$-amino-3-hidroxi-5-metilo-4isoxazolpropiónico antiglutamato tipo 1 y 2, anticuerpos contra la proteína asociada a contactina-2 (CASPR2), anticuerpos contra el receptor del ácido $\gamma$-aminobutírico de tipo $\mathrm{B}\left(\mathrm{GABA}_{\mathrm{B}}\right)$, anticuerpos contra la proteína 1 inactivada del glioma rica en leucina (LGI1). LCR: líquido cefalorraquídeo; PCR: reacción en cadena de la polimerasa; PDRG: pruebas de diagnóstico rápido de la gripe. 
hiperintenso en las imágenes FLAIR ponderadas en T2 e isointenso con difusión restringida en las imágenes ponderadas en T1 sin realce del contraste (Figura 1A-D1). Por lo tanto, se le diagnosticó encefalitis/ encefalopatía leve con lesión reversible del esplenio del cuerpo calloso (MERS). Las alteraciones en la RM fueron similares en 3 casos (pacientes 2, 3 y 7). En todos se observó tumefacción de ambos núcleos caudados e hiperintensidad en los núcleos lenticulares en las imágenes FLAIR ponderadas en T2 (Figura 1A$A 1, B 1, C 1)$, y esta lesión tenía difusión restringida con un aumento de la intensidad de señal en los mapas ADC. El paciente 10 tenía alteraciones de la señal en la protuberancia anular, el mesencéfalo, el bulbo raquídeo y los pedúnculos cerebelosos con hiperintensidad en las imágenes FLAIR ponderadas en T2 y difusión restringida con una disminución de la intensidad de señal en los mapas ADC de la protuberancia anular (Figura 1A-E1). En la RM de la paciente 11, se observaron alteraciones hemorrágicas en el tálamo, el mesencéfalo y el cerebelo en ambos hemisferios. En las imágenes FLAIR ponderadas en $\mathrm{T} 2$, se observó hiperintensidad en el tálamo, los núcleos basales, el mesencéfalo, la protuberancia anular y la sustancia blanca periventricular en ambos hemisferios (Figura 1AF1). En las imágenes del mapa ADC ponderadas por difusión, se observó difusión restringida en el tálamo, el asta posterior de la cápsula interna y la sustancia blanca periventricular frontoparietal en ambos hemisferios. En los estudios de RM del paciente 5, se observó expansión de los giros cerebrales y edema en la región frontotemporoparietal izquierda en los estudios anteriores a la administración de contraste con hiperintensidad en las imágenes FLAIR ponderadas en T2. Además, se detectó realce leptomeníngeo en las imágenes FLAIR posteriores a la administración de contraste. En el caso de la paciente 13, se observó realce del contraste en el cono medular y las raíces de los nervios de la cola de caballo. Las RM de seguimiento revelaron una mejoría (Figura 1B).

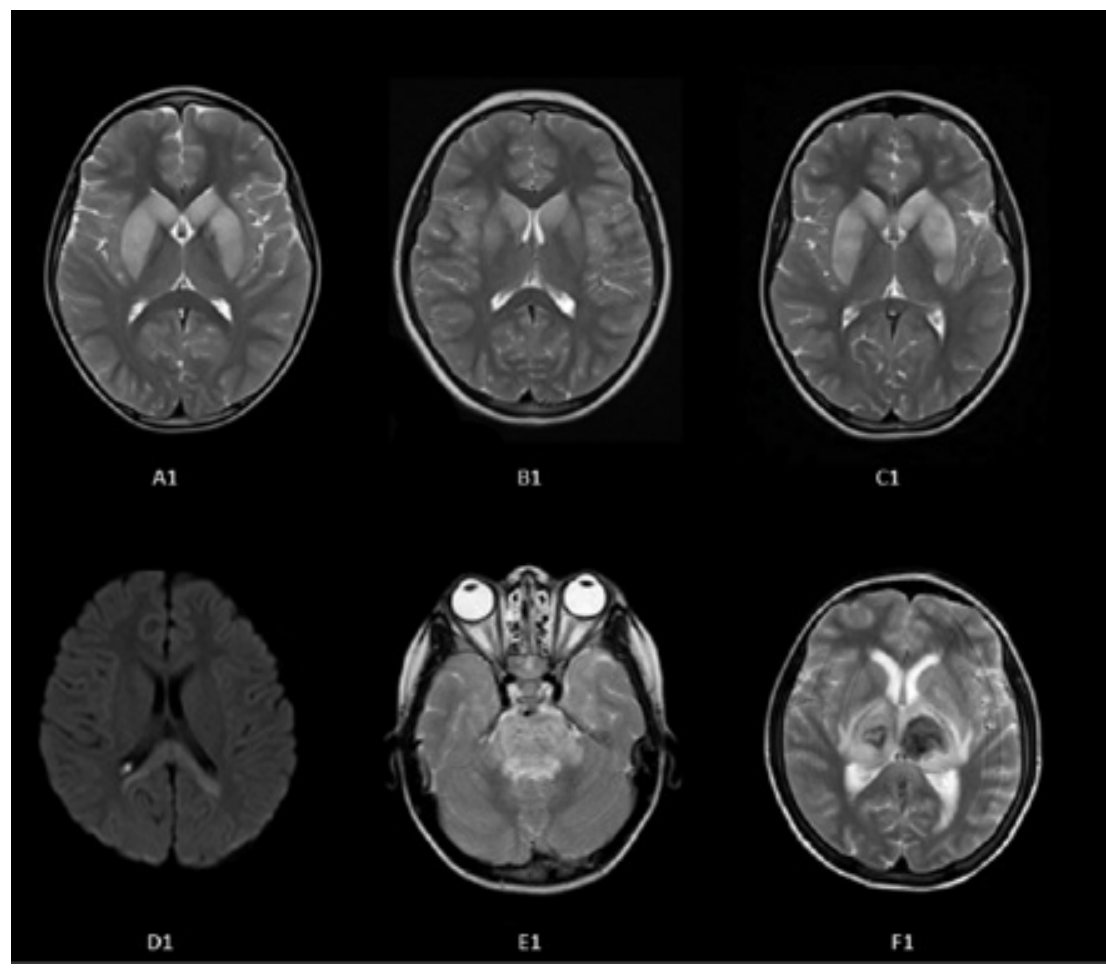

Hinchazón en ambos núcleos caudados e hiperintensidad en los núcleos lenticulares en las imágenes FLAIR ponderadas en T2 (A1, B1, C1), alteraciones de la señal en el esplenio del cuerpo calloso, con hiperintensidad en las imágenes FLAIR ponderadas en T2 (D1). Hiperintensidad en la protuberancia anular y el pedúnculo cerebeloso en las imágenes FLAIR ponderadas en T2 (E1), hiperintensidad y alteraciones hemorrágicas en el tálamo, en ambos hemisferios, en las imágenes FLAIR ponderadas en T2 (F1). Los pacientes 1, 6, 8, 9 y 12 tuvieron resultados normales en la RM. 


\section{Tratamiento}

Se administraron aciclovir y oseltamivir como tratamiento antiviral hasta obtener los resultados microbiológicos. Once pacientes recibieron un tratamiento antiviral en el transcurso de las primeras 12 horas. El paciente 5 fue trasladado a nuestro hospital y se le administró el tratamiento antiviral 72 horas después de que solicitó atención médica. Se administró metilprednisolona (MP) en inyección intravenosa a 11 pacientes con

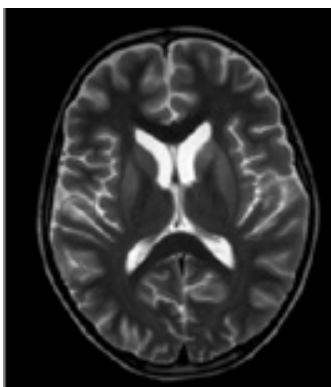

A2

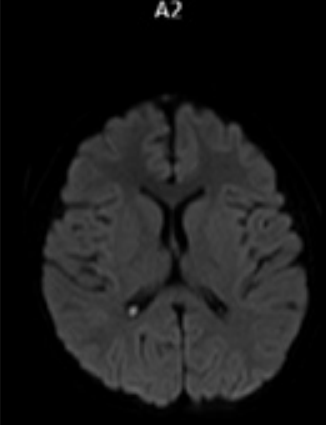

D2

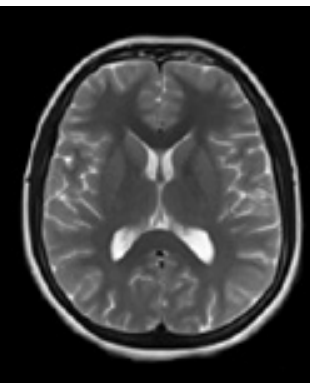

B2

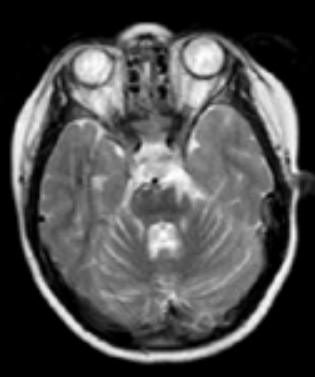

E2

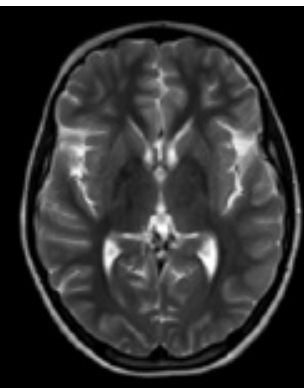

c2

Mejoría después del tratamiento (A2-E2). No se realizó la RM de seguimiento de la paciente 11 debido a la pandemia de COVID-19.

TABLA 3. Tratamiento de los pacientes con encefalitis asociada a la gripe

\begin{tabular}{lcccccc}
\hline $\begin{array}{l}\text { Número } \\
\text { de caso }\end{array}$ & Oseltamivir & Aciclovir & $\begin{array}{c}\text { MP en inyección } \\
\text { intravenosa }\end{array}$ & $\begin{array}{c}\text { IgIV } \\
\mathbf{( 2} \mathbf{~ g} / \mathbf{k g})\end{array}$ & Plasmaféresis & Antiepilépticos \\
\hline 1 & + & + & $+/ 5$ días & - & - & Levetiracetam \\
2 & + & $+/ 5$ días & $+/ 4$ días & $+/ 7$ días & - \\
3 & + & $+/ 5$ días & $+/ 4$ días & $+/ 7$ días & - \\
4 & + & + & - & - & - \\
5 & + & + & $+/ 5$ días & $+/ 4$ días & - & Levetiracetam $/$ \\
6 & + & + & $+/ 5$ días & - & - & alproico \\
7 & + & + & $+/ 5$ días & $+/ 4$ días & $+/ 7$ días & Levetiracetam \\
8 & + & + & - & - & - & Fenitoína \\
9 & + & + & $+/ 5$ días & $+/ 4$ días & $+/ 7$ días & Levetiracetam \\
10 & + & + & $+/ 5$ días & $+/ 4$ días & $+/ 7$ días & Levetiracetam \\
11 & + & + & $+/ 5$ días & $+/ 4$ días & $+/ 7$ días & Levetiracetam \\
12 & + & - & - & $+/ 4$ días & - & -
\end{tabular}

MP: metilprednisolona; IgIV: inmunoglobulina intravenosa. 
un tiempo de inicio de 18,5 h. Se administró inmunoglobulina intravenosa (IgIV) en 7 casos, que estaban en fases más avanzadas y tenían alteraciones más graves en la RM. El paciente al que se le diagnosticó síndrome de GuillainBarré también recibió IgIV. Además, se realizó plasmaféresis a 6 pacientes que tuvieron una respuesta parcial al tratamiento con MP en inyección intravenosa y con IgIV. Se administró una solución salina hipertónica para tratar los edemas. Se administró un tratamiento anticonvulsivo a 6 pacientes que tuvieron convulsiones y a 2 pacientes de forma preventiva (Tabla 3).

\section{Abordaje y resultados}

Once pacientes debieron permanecer en la UCIP durante una media de 16,3 días (mín.: 3 días-máx.: 44 días). Todos estos pacientes recibieron el alta hospitalaria con un puntaje GOSE medio de 7,3 (mín.: 5-máx.: 8) y se los contactó para un seguimiento mensual durante 9 meses. Los pacientes 5 y 11 presentaron epilepsia y requirieron un tratamiento anticonvulsivo (levetiracetam y ácido valproico). Además, los pacientes 10 y 11 tuvieron cambios de comportamiento, un retroceso de las funciones cognitivas y dificultad para caminar (Tabla 1). Seis pacientes recibieron fisioterapia tras el alta.

\section{DISCUSIÓN}

La gripe es una de las causas más frecuentes de encefalitis/ encefalopatía aguda en pediatría. La encefalitis/ encefalopatía asociada a la gripe se observó en todas las edades, desde la lactancia hasta la pubertad. ${ }^{4}$

Las epidemias de gripe tienen un patrón estacional característico, con un pico de incidencia en otoño e invierno. ${ }^{6}$ Las vacunas inactivadas y atenuadas con microbios vivos reducen el riesgo de infección gripal en los niños. ${ }^{7}$ No obstante, no hay certeza de que exista una relación entre la vacunación contra la gripe y la protección contra las complicaciones neurológicas. ${ }^{3}$

El mecanismo subyacente de la encefalitis asociada a la gripe es controvertido. Hay diferentes opiniones sobre la patogenia; la invasión viral, determinados acervos genéticos y la tormenta de citocinas podrían afectar la función del sistema nervioso central (SNC) y dañar el tejido del SNC. ${ }^{8}$ Fujimoto y cols., informaron que se detectó el ARN del virus de la gripe en el LCR. ${ }^{9}$ Por el contrario, en otros informes, solo unos pocos pacientes tuvieron resultados positivos de ARN viral en el LCR. ${ }^{8}$ En nuestros pacientes, solo se detectó ARN viral en las muestras de los cultivos de exudado nasal. Con nuestros datos, abordamos el tratamiento de nuestros pacientes como una enfermedad inmunitaria, y no como la infección principal.

Los cambios de neuroimagenología de la encefalitis / encefalopatía asociada a la gripe incluyen alteraciones de la señal de la sustancia blanca cortical y subcortical, edema localizado o generalizado y lesiones multifocales simétricas bilaterales en el tálamo y la médula del cerebelo. ${ }^{10}$ A continuación, se presentan diferentes diagnósticos clínicos y de neuroimagenología: encefalopatía necrotizante aguda (ENA), síndrome de encefalopatía posterior reversible (SEPR), encefalomielitis aguda diseminada (EMAD), MERS, meningoencefalitis, encefalopatía aguda con convulsiones bifásicas y difusión reducida tardía (AESD), síndrome de choque hemorrágico y encefalopatía (HSES). ${ }^{11,12}$ Las RM de los pacientes 4, 5, 10, 11 y 13 tuvieron similitudes con lo expuesto en la bibliografía. En la RM de los pacientes 2, 3 y 7, se observó la afectación del núcleo caudado y el putamen en ambos hemisferios. La afectación aislada bilateral del núcleo caudado y del putamen no se observa con frecuencia en la bibliografía existente. ${ }^{2,4,12,13}$

No existe un tratamiento específico para la encefalitis asociada a la gripe y hay diferentes formas de tratarla, que incluyen el tratamiento antiviral, corticoesteroides en dosis altas, IgIV y plasmaféresis. ${ }^{1}$ La eficacia del oseltamivir con resultados favorables no es absoluta; no obstante, en las pautas de la Sociedad de Enfermedades Infecciosas de Estados Unidos, se recomienda el uso de oseltamivir, y algunas series aportaron datos que lo respaldan. ${ }^{5,14}$ La MP en inyección intravenosa, la IgIV y la plasmaféresis constituyen la inmunoterapia de elección para la encefalitis autoinmunitaria. ${ }^{15}$ Los mecanismos inmunitarios se consideran la patogenia subyacente de la encefalitis asociada a la gripe; por lo tanto, la MP en inyección intravenosa y la IgIV son las opciones terapéuticas más frecuentes. ${ }^{4}$

Se informó que entre el $21 \%$ y el $45 \%$ de los pacientes que sobreviven a la encefalitis asociada a la gripe tienen deficiencias neurológicas. ${ }^{1,3}$ Aún no se han determinado los factores pronósticos específicos de la encefalitis asociada a la gripe; ${ }^{3}$ el diagnóstico temprano fue el factor más relevante en nuestros casos. La RM es fundamental para lograr un diagnóstico y un tratamiento tempranos. Los hallazgos de la RM de la paciente 11 eran 
los más graves, con características clínicas potencialmente mortales. Se le dio el alta con un puntaje GOSE de $5 \mathrm{y}$, durante el seguimiento, logró caminar con apoyo. El paciente 5 recibió el tratamiento más tarde que los demás, su puntaje GOSE fue bajo (6) y tuvo secuelas neurológicas. Los puntajes GOSE de los demás pacientes fueron altos (GOSE 7, 8), y estos no tuvieron secuelas durante el seguimiento. Creemos que la detección temprana y un tratamiento intensivo y temprano son importantes para la morbimortalidad en esta experiencia.

En este artículo, se presentaron diferentes complicaciones neurológicas de la gripe como hallazgos de la RM y características clínicas asociadas. La detección de las complicaciones neurológicas de la gripe es difícil, sobre todo cuando el cuadro clínico inicial no es típico. Los estudios de RM son sumamente importantes para lograr un diagnóstico y un tratamiento tempranos. La afectación de los núcleos caudados y del putamen en la RM no es frecuente en los casos de encefalitis asociada a la gripe. No obstante, durante la temporada gripal, se debe tener especialmente en cuenta esta afectación mediante exámenes clínicos y análisis de laboratorio y se debe iniciar el tratamiento lo antes posible.

\section{Agradecimientos}

Agradecemos la ardua labor del Departamento de Enfermedades Infecciosas Pediátricas y de la Unidad de Cuidados Intensivos Pediátricos.

\section{REFERENCIAS}

1. Paksu MS, Aslan K, Kendirli T, Akyildiz BN, et al. Neuroinfluenza:evaluation of seasonal influenza associated severeneurological complications in children (a multicenter study). Childs Nerv Syst. 2018; 34(2):335-47.

2. DadakM, Pul R, Lanfermann H, Hartmann H, etal. Varying Patterns of CNS Imaging in Influenza A Encephalopathy in Childhood. Clin Neuroradiol. 2019; 30(2):243-9.

3. Britton PN, Blyth CC, Macartney K, Dale RC, et al. The Spectrum and Burden of Influenza-Associated Neurological Disease in Children: Combined Encephalitis and Influenza Sentinel Site Surveillance From Australia, 2013-2015. Clin Infect Dis. 2017; 65(4):653-60.

4. Chen LW, Teng CK, Tsai YS, Wang JN, et al. Influenzaassociated neurological complications during 2014-2017 in Taiwan. Brain Dev. 2018; 40(9):799-806.

5. Goenka A, MichaelBD, LedgerE, HartIJ, et al. Neurological manifestations of influenza infection in children and adults: results of a National British Surveillance Study. Clin Infect Dis. 2014; 58(6):775-84.

6. Gómez-Barroso D, León-Gómez I, Delgado-Sanz C, Larrauri A. Climatic Factors and Influenza Transmission, Spain, 2010-2015. Int J Environ Res Public Health. 2017; 14(12):1469.

7. Jefferson T, Rivetti A, Di Pietrantonj C, Demicheli V. Vaccines for preventing influenza in healthy children. Cochrane Database Syst Rev. 2018; 2(2):CD004879.

8. Wang GF, Li W, Li K. Acute encephalopathy and encephalitis caused by influenza virus infection. Curr Opin Neurol. 2010; 23(3):305-11.

9. Fujimoto S, Kobayashi M, Uemura O, Iwasa M, et al. PCR on cerebrospinal fluid to show influenza-associated acute encephalopathy or encephalitis. Lancet. 1998; 352(9131):873-5.

10. SuranaP, TangS, McDougall M, Tong CY, etal.Neurological complications of pandemic influenza A H1N1 2009 infection: European case series and review. Eur J Pediatr. 2011; 170(8):1007-15.

11. Wilking AN, Elliott E, García MN, Murray KO, Munoz FM. Central nervous system manifestations in pediatric patients with influenza A H1N1 infection during the 2009 pandemic. Pediatr Neurol. 2014; 51(3):370-6.

12. Amin R, Ford-Jones E, Richardson SE, MacGregor D, et al. Acute childhood encephalitis and encephalopathy associated with influenza: a prospective 11-year review. Pediatr Infect Dis J. 2008; 27(5):390-5.

13. Britton PN, Dale RC, Blyth CC, Macartney K, etal. Influenzaassociated Encephalitis / Encephalopathy Identified by the Australian Childhood Encephalitis Study 2013-2015. Pediatr Infect Dis J. 2017; 36(11):1021-6.

14. Tunkel AR, Glaser CA, Bloch KC, Sejvar JJ, et al. The management of encephalitis: clinical practice guidelines by the Infectious Diseases Society of America. Clin Infect Dis. 2008; 47(3):303-27.

15. Shin YW, Lee ST, Park KI, Jung KH, et al. Treatment strategies for autoimmune encephalitis. Ther Adv Neurol Disord. 2018; 11:1756285617722347. 